EPJ Web of Conferences 71, 00003 (2014)

DOI: $10.1051 /$ epjconf / 20147100003

(C) Owned by the authors, published by EDP Sciences, 2014

\title{
Report from ECFA
}

\author{
Călin Alexa ${ }^{1, a}$ \\ ${ }^{1}$ IFIN-HH, Particle Physics Department, Magurele-Bucharest 077125, România
}

\begin{abstract}
The European Committee for Future Accelerators was founded in 1963 with one of its main activities being to monitor and support the development of particle physics in the CERN member countries through regular visits. Information about the mission of the Committee and its recent activities will be provided. With the involvement of ECFA the Update of the European Strategy for Particle Physics was developed and approved by CERN Council in May 30, 2013. A summary of the recommendations of the Strategy Update will be presented.
\end{abstract}

\section{ECFA terms of reference}

The European Committee for Future Accelerators (ECFA) [1] aims are to elaborate long-range planning of European high-energy large-scale facilities, to oversee the equilibrium between the roles of international and national laboratories and university institutes, to ensure adequate conditions for research and a just and equitable sharing of facilities between physicists, irrespective of nationality and origin [2].

Monitoring the development of particle physics in the CERN member countries through regular visits is one of the ECFA's main activities. The visits are made by the members of the Restricted ECFA (RECFA) that consists of one representative per country, the Director General and the Research Director of CERN, the Scientific Secretary of the Strategy Session of CERN Council, the DESY Director in charge of High Energy Physics and Astroparticle Physics, and the Director of the INFN Frascati Laboratory. The purpose of these visits, which are made to all member states of CERN in rotation, is to assess particle physics and related disciplines in that country and to suggest improvements. The recommendations are made both to the physics community and to the government. Monitoring through country visits of the ongoing implementation of the European Strategy for Particle Physics is a very important activity of the Committee.

ECFA may organize or sponsor conferences, may also propose, support or oversee study groups and review large scale facilities proposals.

\section{Update of the European Strategy for Particle Physics}

The first European Strategy for Particle Physics [3], approved by the CERN Council on 14th July2006 in a special meeting held in Lisbon, contained 17 statements on scientific and organizational matters.

\footnotetext{
${ }^{\text {a }}$ Scientific Secretary of ECFA. e-mail: Calin.Alexa@nipne.ro
} 
If the highest priority was the completion of the LHC machine, the strategy also recommends advanced accelerator studies for the CLIC and high performance magnets, ILC design and the participation in a global neutrino program. It was stated that the Council will define and update the strategy based on proposals and observations from a dedicated scientific body that shall be established for this purpose.

The update process of the European Strategy for Particle Physics started officially in September 2011 by the Council establishing the Strategy Group and the Preparatory Group.

Representatives from each CERN member state and the directors of the major European laboratories formed the Strategy Group. The strategy secretariat, consisting of the Scientific Secretary for Strategy Session of CERN Council, the chairpersons of the CERN Scientific Policy Committee (SPC) and the European Committee for Future Accelerators (ECFA), the representative of the European laboratory directors, and a Scientific Assistant, has coordinated the upgrade. Representatives from the candidates for accession and associate member states, from observer states, the director of JINR, Dubna, as well as representatives from EU, APPEC [4], and the chairpersons from FALC [5], ESFRI [6] and NuPECC [7] were invited to attend the Strategy Group meetings.

The Preparatory Group, consisting of eight members from ECFA and SPC, the scientific secretary, and one representative from Asia and one from the Americas, had the task to prepare the background material for the Strategy Group and to organize an open symposium and the drafting session.

In February 2012, the call for scientific input from the community was launched and in September 2012 an Open Symposium was organized by the Preparatory Group in Kracow [8]. Based on the information from the written contributions and the presentations and discussions during the open symposium the Preparatory Group produced the Scientific Briefing Book [9]. In January 2013 the Strategy Group met in Erice and a draft of the Update of the European Strategy for particle physics was agreed and submitted to CERN Council. In March 2013, the CERN Council agreed on the final version and the Update of the European Strategy was formally adopted on 30 May 2013 during a special session of Council in Brussels.

\section{Summary of the European Strategy for Particle Physics}

The approved Update of the European Strategy is divided in seven sections, each of them contains subsections labeled (a)-(q). The sections are Preamble, General Issues (a)-(b), High-priority largescale scientific activities (c)-(f), Other scientific activities essential to the particle physics programme $(\mathrm{g})-(\mathrm{k})$, Organisational issues (l)-(m), Wider impact of particle physics (n)-(p) and Concluding recommendations (q).

We will not reproduce the entire European Strategy for Particle Physics strategy statement paper [10]. The reader is invited to consult the official site of the European Strategy for Particle Physics [3] and a summary of the main points of the approved European Strategy Update [11]. We will rather present a summary of the requirements of the strategy update.

a) Europe should preserve the European organizational model for particle physics in order to keep its leading role, sustaining the success of particle physics and the benefits it brings to the wider society.

b) The European Strategy takes into account the worldwide particle physics landscape and developments in related fields and should continue to do so.

c) Europe's top priority should be the exploitation of the full potential of the LHC, including the high-luminosity upgrade of the machine and detectors with a view to collecting ten times more data than in the initial design, by around 2030 . 
d) CERN should undertake design studies for accelerator projects in a global context, with emphasis on proton-proton and electron-positron high-energy frontier machines. These design studies should be coupled to a vigorous accelerator R\&D programme, including high-field magnets and high-gradient accelerating structures, in collaboration with national institutes, laboratories and universities worldwide.

e) Europe looks forward to a proposal from Japan to discuss a possible participation in the International Linear Collider (ILC).

f) CERN should develop a neutrino programme to pave the way for a substantial European role in future long-baseline experiments. Europe should explore the possibility of major participation in leading long-baseline neutrino projects in the US and Japan.

g) Europe should support a diverse, vibrant theoretical physics programme, ranging from abstract to applied topics, in close collaboration with experiments and extending to neighbouring fields such as astroparticle physics and cosmology. Such support should extend also to high-performance computing and software development.

h) Experiments in Europe with unique reach should be supported, as well as participation in experiments in other regions of the world.

i) Detector R\&D programmes should be supported strongly at CERN, national institutes, laboratories and universities. Infrastructure and engineering capabilities for the $R \& D$ programme and construction of large detectors, as well as infrastructures for data analysis, data preservation and distributed dataintensive computing should be maintained and further developed.

j) In the coming years, CERN should seek a closer collaboration with ApPEC on detector R\&D with a view to maintaining the community's capability for unique projects in this field.

k) The CERN Laboratory should maintain its capability to perform unique experiments. CERN should continue to work with NuPECC on topics of mutual interest.

1) CERN should be the framework within which to organise a global particle physics accelerator project in Europe, and should also be the leading European partner in global particle physics accelerator projects elsewhere. Possible additional contributions to such projects from CERN's Member and Associate Member States in Europe should be coordinated with CERN.

m) CERN and the particle physics community should strengthen their relations with the European Commission in order to participate further in the development of the European Research Area.

n) Outreach and communication in particle physics should receive adequate funding and be recognised as a central component of the scientific activity. EPPCN and IPPOG should both report regularly to the Council.

o) HEPTech should pursue and amplify its efforts and continue reporting regularly to the Council.

p) CERN, together with national funding agencies, institutes, laboratories and universities, should continue supporting and further develop coordinated programmes for education and training.

q) Updates of the European Strategy should continue to be undertaken according to the principles applied on the present occasion. The organisational framework for the Council Sessions dealing with European Strategy matters and the mechanism for implementation and follow-up of the Strategy should be revisited in the light of the experience gained since 2006. 
It is worth noting that the Strategy Update recommend periodic updates at intervals of about 5 years. Also recommended was to revisit the organizational framework in which the CERN Council is dealing with European strategy matters.

\section{References}

[1] The European Committee for Future Accelerators http://ecfa.web.cern.ch/ecfa/en/Welcome.html

[2] ECFA terms of reference http://ecfa.web.cern.ch/ecfa/en/termsofref.html

[3] The European Strategy Session of Council http://council.web.cern.ch/council/en/EuropeanStrategy/ESParticlePhysics.html

[4] AstroParticle Physics European Consortium http://www.appec.org/

[5] Funding Agencies for Large Colliders http://www.falchep.org/

[6] European Strategy Forum on Research Infrastructures http://ec.europa.eu/research/infrastructures

[7] Nuclear Physics European Collaboration Committee http://www.nupecc.org/

[8] European Strategy for Particle Physics, 10-12 Sept. 2012, Krakow, Poland http://espp2012.ifj.edu.pl

[9] The European Strategy for Particle Physics - Scientific Briefing Book http://europeanstrategygroup.web.cern.ch/europeanstrategygroup/Briefing_book.pdf

[10] Strategy Statement paper http://council.web.cern.ch/council/en/EuropeanStrategy/esc-e-106.pdf

[11] M. Krammer, The update of the European strategy for particle physics, Phys. Scr. T158 (2013) 014019 